\title{
Publisher's Note: Anomalous Conductances in an Ultracold Quantum Wire [Phys. Rev. Lett. 117, 255302 (2016)]
}

M. Kanász-Nagy, L. Glazman, T. Esslinger, and E. A. Demler

(Q) (Received 9 January 2019; published 23 January 2019)

DOI: 10.1103/PhysRevLett.122.039901

This paper was published online on 16 December 2016 with an error in a support provider and a contract number in the Acknowledgments on page 5. On page 5, the fourth sentence in the Acknowledgments should read as "L. G. was supported by DOE contract DE-FG02- 08ER46482." The Acknowledgments have been corrected as of 16 January 2019. The Acknowledgments are incorrect in the printed version of the journal. 